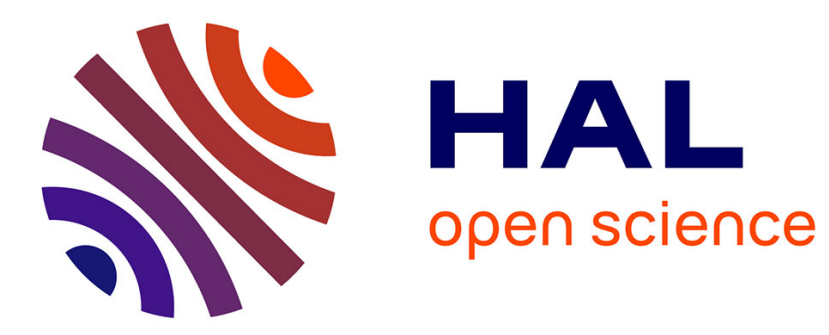

\title{
Gouvernance et performance des pôles de PME
}

Rachel Bocquet, Caroline Mothe

\section{To cite this version:}

Rachel Bocquet, Caroline Mothe. Gouvernance et performance des pôles de PME. Revue Française de Gestion, 2009, 10 (190), pp.101-122. hal-01292077

\section{HAL Id: hal-01292077 \\ https://hal.science/hal-01292077}

Submitted on 22 Mar 2016

HAL is a multi-disciplinary open access archive for the deposit and dissemination of scientific research documents, whether they are published or not. The documents may come from teaching and research institutions in France or abroad, or from public or private research centers.
L'archive ouverte pluridisciplinaire $\mathbf{H A L}$, est destinée au dépôt et à la diffusion de documents scientifiques de niveau recherche, publiés ou non, émanant des établissements d'enseignement et de recherche français ou étrangers, des laboratoires publics ou privés. 


\title{
Gouvernance et performance des pôles de PME
}

\author{
Rachel Bocquet \\ IREGE, Université de Savoie \\ rachel.bocquet@univ-savoie.fr \\ Caroline Mothe $(*)$ \\ IREGE, Université de Savoie \\ caroline.mothe@univ-savoie.fr
}

\section{Résumé}

Si la littérature a largement traité du rôle des déterminants structurels de la performance des formes agglomérées territorialisées, le lien entre gouvernance et performance n'a été que peu appréhendé. Nous tentons de combler ce vide en cernant les caractéristiques de la gouvernance des pôles de compétitivité français. L'enquête qualitative sur Arve-Industries, pôle à forte dominante PME, fait ressortir l'importance de l'implication des membres de la gouvernance comme déterminant clé des performances collective et individuelle. Par ailleurs, la gouvernance doit s'adapter à l'évolution du pôle, notamment passer par une formalisation lorsque le pôle se situe en phase de croissance.

Mots clés : Gouvernance, PME, Performance, Pôle de compétitivité

JEL : 018 ; 032

(*) pour toute correspondance

Nous remercions l'AED (Agence Economique Départementale de Haute-Savoie), Thésame (Centre Européen d'Entreprise et d'Innovation), les membres de la gouvernance du pôle ArveIndustries et les deux évaluateurs pour leurs commentaires constructifs. 
Sur 4611 entreprises participant aux pôles de compétitivité lancés en 2005 par le gouvernement français, 3905 (soit $85 \%$ ) sont des $\mathrm{PME}^{1}$. Après trois ans d'existence et face à la grande variété des performances atteintes ${ }^{2}$, la question des déterminants de la performance d'un pôle s'avère cruciale. De même, le problème de la définition de la performance reste posé. En effet, peut-on attendre la même performance d'un pôle à forte dominante PME que d'un pôle regroupant une majorité de grandes entreprises ? Peut-on retenir une définition unique de la performance indépendamment d'une spécificité de nature?

La littérature sur les formes territorialisées fournit une première réponse à ce questionnement sous l'angle des seuls éléments structurels. Dans cette perspective, la performance des pôles de compétitivité dépendrait étroitement de leur nature hybride entre « district industriel» et «cluster». Sans négliger l'impact de ces éléments structurels, nous tentons, dans la lignée des quelques recherches récentes sur le lien entre gouvernance et performance des formes territorialisées, de comprendre l'impact de la gouvernance sur la/les performance(s). L'introduction de la gouvernance comme facteur de performance nous semble particulièrement justifié dans le cas des pôles à forte dominante PME.

L'objectif de la première partie est de construire une grille d'analyse qui permette de rendre compte de la performance de cette forme organisationnelle territoriale a priori hybride. Les travaux empiriques sur les pôles mettent l'accent sur deux types de spécificités susceptibles d'influencer leur performance - et celle des entreprises qui les composent. La première dépendrait de la forme d'agglomération spatiale. Il est aujourd'hui largement admis que la notion de pôle peut englober une variété de formes d'agglomération spatiale des activités, qui diffèrent en termes de performance attendue. En se basant sur les recherches et les typologies existantes, nous montrons en quoi la performance d'un pôle «proche d'un district industriel » n'est pas la même que celle que l'on pourrait attendre d'un pôle «proche d'un cluster anglosaxon ou à la Porter ». La seconde spécificité associée au pôle à forte dominante PME réside dans le rôle crucial que jouerait la gouvernance à l'échelle locale. En effet, les études empiriques centrées sur les agglomérations de PME montrent que celle-ci est un déterminant clé de leur performance et ce, quel que soit leur type (district, cluster, pôle).

\footnotetext{
${ }^{1}$ http://www.competitivite.gouv.fr/IMG/pdf/TdB_TousPoles.pdf (15/09/2008). Les PME sont définies comme des entreprises de moins de 250 salariés, l'effectif étant le seul critère utilisé, sans que l'appartenance à un groupe ne soit prise en compte.

${ }^{2}$ Les résultats de l'évaluation des 71 pôles confirment avec force cet aspect : 39 pôles ont atteint les objectifs, 19 pôles les ont atteint partiellement, 13 pôles pourraient tirer partie d'une reconfiguration en profondeur (cf. rapport de synthèse, BCG, CMI, 18 juin 2008).
} 
Dans la seconde partie, nous proposons de contrôler si ces déterminants de la performance sont applicables à un pôle à forte dominante de PME comme Arve-Industries. Labellisé depuis deux ans, ce pôle est composé à 90\% de PME. La méthodologie qualitative exploratoire, basée sur un guide d'entretien semi-directif, a conduit à interroger les membres de la gouvernance (19 au total). Le statut multidimensionnel du concept de performance est saisi en mettant en avant les mesures clés des performances individuelle et collective retenues par les répondants. L'analyse de contenu des entretiens permet d'une part, d'identifier les spécificités de la performance de ce type de pôle et, d'autre part, de mettre en évidence l'impact de la gouvernance sur ladite performance. Notre discussion porte à la fois sur les spécificités de la performance d'un pôle de PME et sur la gouvernance comme facteur de performance.

\section{La performance des pôles de compétitivité à forte dominante PME : un modèle de performance « hybride »?}

Pour construire une grille d'analyse de la performance adaptée au pôle de compétitivité à forte dominante PME, il convient dans une premier temps de saisir sa nature et ses spécificités par rapport au district industriel «à la Becattini » (1981) et au cluster «à la Porter» (2000, 2004). $\mathrm{Au}$ plan théorique, ces deux formes d'agglomération d'activités correspondent bien à deux configurations qui diffèrent par nature. A la différence des typologies récentes, nous ne cherchons pas à positionner le pôle par rapport à d'autres formes nées de politiques publiques volontaristes telles que le SPL (Système Productif Local), le technopôle ou encore les milieux innovateurs. Nous privilégions les formes dites «pures »- et bien stabilisées théoriquement pour identifier les principaux déterminants de la performance qui leur est associée et la nature de la performance attendue aux niveaux collectif (performance du pôle) et individuel (performance des entreprises du pôle). Dans un second temps, nous enrichissons notre grille d'analyse de la performance en mobilisant la littérature consacrée plus spécifiquement aux agglomérations spatiales de PME. Ceci nous permet de compléter la liste des déterminants en introduisant le rôle que peut jouer la gouvernance sur la performance des pôles de PME.

Nous verrons dans quelle mesure ces éléments conditionnent deux types de performance distinctes (1.1). Puis nous complétons la description des deux formes typiques en discutant du rôle que peut jouer la gouvernance pour accroître la performance d'un pôle de PME (1.2).

\subsection{Le district industriel et le cluster : deux idéaux-types de performance}

La distinction opérée entre les deux formes d'agglomération spatiale que sont le district industriel «à la Becattini » (1981) et le cluster « à la Porter»(2000, 2004) est relativement 
classique. Toutefois, si de nombreux travaux, y compris les typologies les plus récentes, (Defélix et al. 2006, Carluer, 2006, Calmé, Chabault, 2007) ont bien commenté les spécificités associées à ces deux configurations, rares sont ceux qui débouchent sur une lecture de la performance associée à ces deux formes.

\subsubsection{Le district industriel : la performance est « dans l'air »}

Le district industriel (Becattini, 1981) est un modèle d'agglomération spatiale des activités basé sur une forte division du travail entre de petites entreprises spécialisées sur différentes étapes d'un même processus productif. Cet espace productif n'est pas limité aux relations industrielles mais intègre de multiples relations entre les entreprises, la communauté locale et le marché. La performance du système, en l'absence d'une entreprise leader, dépend étroitement de la force du potentiel institutionnel (par définition endogène). Ce potentiel est associé à des facteurs historiques et socioculturels qui participent à la création d'une culture commune et d'un climat de confiance et de réciprocité. Comme le notent Moulaert et Sekia (2003), «cette forme hybride d'organisation combinant compétition et coopération, relations institutionnelles formelles et informelles, ne peut pas être comprise sans mettre en valeur le rôle des facteurs historiques et socio-économiques, clés pour le succès du district » (p. 291). La coopération, établie sur un plan essentiellement technique, passe par des relations non marchandes et le plus souvent informelles entre des individus (Grossetti, 2004). La concurrence sur les prix reste très intense compte tenu de l'appartenance des entreprises à un même secteur, voire à une même activité.

Les avantages liés à cet espace dynamique sont incarnés dans les externalités d'agglomération, pécuniaires et de communication, dont peuvent bénéficier les PME qui y participent. Les externalités pécuniaires, à la différence des externalités de communication, n'exigent pas d'interactions directes entre les PME. Elles résultent des stratégies de localisation des entreprises en un même lieu, ce qui génère des gains monétaires liés à la disponibilité d'une main d'œuvre qualifiée, à la qualité des infrastructures, ou encore au soutien des institutions locales. Les externalités de communication, quant à elles, s'apparentent à des bénéfices liés à un processus de rencontres et d'échanges de connaissances entre des acteurs géographiquement proches. Dans ces conditions, les innovations présentent un caractère spontané. Elles sont produites à un coût plus faible impliquant des surplus plus élevés (Antonelli, 2000). La mise en perspective de ces deux types d'externalités comme piliers de la performance conduit néanmoins à deux critiques.

La première a trait à la dimension «localiste » de la performance dont il est question. La dynamique locale est déterminée par les frontières géographiques du territoire - qui sont, $a$ 
priori, données. La performance du territoire est vue comme exogène aux acteurs, ces derniers ne participant pas activement à la dynamique locale. Une telle conception conduit à surestimer le rôle des effets bénéfiques de la proximité géographique (Tallman et al., 2004). Alberti (2001) soutient également que cette forme territorialisée s'accompagne d'un déficit de dynamique collective malgré les bénéfices attendus de la co-localisation. Les obstacles tels que les comportements opportunistes, les conflits d'intérêt et l'individualisme des acteurs seraient même aggravés par la proximité géographique (Gundolf et al., 2007).

La seconde critique concerne la nature de l'innovation. Le district, du moins à l'origine, est d'abord un mode d'organisation local de la production basé sur une forte division du travail entre des petites entreprises familiales issues d'industries traditionnelles. Si certains mettent l'accent sur sa dynamique créatrice (Becattini, 1981), celle-ci doit se traduire par une capacité à accroître et générer des profits à court terme. L'innovation n'est pas a priori un but en soi, mais semble davantage s'apparenter davantage à un produit dérivé de la division du travail.

$\mathrm{Au}$ total, les entreprises n'ont que peu d'emprise sur la performance d'un district, circonscrite aux frontières du territoire. Cette performance est approchée en termes d'accès à un bassin d'emploi dynamique et qualifié, à des infrastructures de qualité et à une base d'informations partagées entre entreprises, les salariés et la communauté locale. Les travaux empiriques sur la performance individuelle des entreprises appartenant à un district sont rares. Aucun ne met en oeuvre une méthodologie comparative - hormis celui de Grando et Belvedere (2005), qui montrent que les entreprises appartenant à un district ne bénéficient que d'un seul type d'avantage : celui de disposer de salariés plus performants dans la mesure où la proximité géographique conduit à une plus grande loyauté des salariés et à un taux d'absentéisme plus faible. Pour les autres mesures de performance (efficience, réactivité, flexibilité, qualité et innovation), les entreprises appartenant à un district n' affichent pas de performance particulière.

\subsubsection{Le cluster « à la Porter »: une performance collective et individuelle maîtrisée}

Le concept de cluster introduit une rupture en défendant l'idée d'une performance construite par des acteurs, non circonscrite aux frontières géographiques du territoire. Contrairement aux districts, définis autour d'une logique d'ancrage territorial et de bassin d'emploi géographiquement limité et restreint (Calmé et Chabaud, 2007 ; Grossetti, 2004), les clusters s'inscrivent dans une logique de développement localisé certes, mais plus fortement axée sur l'innovation. Un cluster est vu comme "un groupe géographiquement proche d'entreprises liées entre elles et d'institutions associées relevant d'un domaine donné, entre lesquelles existent des éléments communs et des complémentarités. Son étendue géographique varie d'une 
seule ville ou d'une région à un pays entier, voire à un réseau de pays voisins » (Porter, 2004, p.207). Si cette définition s'apparente à celle du district, les frontières industrielles et géographiques sont beaucoup plus floues dans la mesure où elles ne peuvent pas être définies a priori (Martin et Sunley, 2003). Il s'agit là d'une véritable rupture : l'espace productif devient un objet socialement construit qui résulte des interactions entre de multiples acteurs privés, publics, locaux et «a-locaux». Cette construction suppose ainsi, au delà de la proximité géographique des acteurs, l'existence de nouvelles formes de proximités (organisationnelle et institutionnelle) qui facilitent l'échange de connaissances entre des acteurs sur la base de valeurs et/ou de routines partagées.

Contrairement à ce qui se passe dans un district, les échanges ne peuvent plus être considérés comme des fuites involontaires de connaissances (externalités de communication). Ils deviennent aussi des échanges volontaires de savoirs organisés dans le cadre d'interactions locales bien maîtrisées par leurs acteurs. Autrement dit, la diffusion localisée des connaissances est en relation avec des pratiques stratégiques et organisationnelles spécifiques qui tendent à en renforcer l'efficacité (Antonelli, 2000 ; Rallet et Torre, 2005). Le concept de cluster conduit ainsi à rejeter l'idée d'une innovation spontanée au profit d'une innovation organisée.

Pour Porter (2000), la capacité d'innovation d'un cluster est étroitement liée à la diversité des membres, qui permet d'éviter la rigidité. Cette diversité est renforcée par le fait que les membres peuvent appartenir à différentes activités et par une libre entrée (et sortie). En effet, la participation à un cluster trop spécialisé conduirait à freiner l'activité d'innovation des membres. Il est donc essentiel de pouvoir bénéficier de nouvelles sources complémentaires de savoirs par l'entrée de nouveaux membres et/ou par la mise en oeuvre de relations privilégiées avec d'autres clusters. Un dosage subtil s'établit ainsi entre concurrence et coopération dans la mesure où la coopération se présente comme un déterminant clé de la performance. En effet, la coopération est source de compétences collectives qui vont au-delà des compétences individuelles de par leur étendue et leur durabilité (Tallman et al., 2004).

La performance d'un cluster (et de ses entreprises, Porter établissant un lien fort entre les deux types de performance) ne repose pas sur un territoire a priori donné. Le cluster s'articule autour de la combinaison de deux dimensions clés : 1) des liens entre des acteurs basés sur des complémentarités et des relations de confiance, 2) des relations de concurrence et de coopération entre des entreprises. Sans renoncer à leur caractère déterminant, Porter va au delà des effets traditionnels associés à la localisation des activités (externalités d'agglomération) et au processus concurrentiel pour intégrer le rôle que peuvent jouer les acteurs eux-mêmes en mettant en oeuvre des schémas organisationnels spécifiques. Outre le fait que la nature de ces 
schémas est souvent imprécise, notons que Porter ne se réfère qu'à quelques clusters spécifiques, comme celui de la Silicon Valley.

Au plan empirique, comme le notent Martin et Sunley (2007), il n'existe pas d'études ayant cherché à tester l'hypothèse clé de Porter, à savoir l'impact positif de l'appartenance à un cluster sur la compétitivité des entreprises. En revanche, de nombreuses recherches permettent de saisir l'influence de certaines des dimensions de la performance. Elles portent essentiellement sur des secteurs dits de haute technologie (nanotechnologie, biotechnologie, informatique, biomédical). Les clusters étudiés sont caractérisés par la présence de grandes entreprises leaders avec une forte capacité d'innovation. L'appartenance à un cluster permet à une entreprise d'avoir accès à des connaissances et à des technologies dont elle n'aurait pas pu bénéficier - ou seulement à des coûts très supérieurs (avec toutefois un risque d'imitation des innovations).

Parallèlement, d'autres résultats identifient des effets négatifs associés à l'appartenance à un cluster, notamment en termes de déséconomies d'agglomération liées à l'augmentation des coûts salariaux, de l'inflation du prix du foncier et à une éventuelle congestion (Martin et Sunley, 2003) qui peut provenir, tout comme les avantages, de la demande ou de l'offre. Ces déséconomies peuvent avoir des conséquences néfastes sur la performance des entreprises. C'est le cas notamment lorsque la sous-traitance locale n'est pas compétitive, obligeant alors les entreprises à aller chercher des inputs ailleurs - ce qui engendre une augmentation des coûts de transaction.

En confrontant ces deux formes d'agglomération spatiale des activités que sont d'une part, le district industriel et d'autre part, le cluster « à la Porter », on aboutit à deux modèles de performance attendue bien distincts (cf. Tableau 1).

\section{Tableau 1 ici}

La principale distinction entre le district et le cluster, qui a un impact sur les autres facteurs, a trait aux types de performance recherchés à l'échelle des entreprises et du pôle. Dans le premier cas (district), on raisonne en termes d'économies de coûts et d'allocation de ressources existantes. Par opposition, la raison d'être du cluster est la production et la diffusion de nouvelles connaissances pour soutenir la dynamique d'innovation. Cette différence est déterminée par plusieurs éléments : la diversité des membres et leur aptitude à innover (intensité et type d'innovation), les barrières à l'entrée et à la sortie, et les modes de coordination entre acteurs.

A cette étape, il convient d'enrichir notre grille d'analyse pour une meilleure adaptation à notre objet d'étude : les pôles à forte dominante PME. Des travaux empiriques récents à la fois sur les districts industriels (Alberti, 2001), les clusters de PME (Carpinetti et al., 2007), les pôles 
à forte dominante PME (Mendez et Bardet, infra ; Gundolf et al,. 2007) convergent pour mettre en exergue le rôle que peut jouer la gouvernance pour des agglomérations de PME. Nous proposons ainsi, à côté des principaux déterminants de structure déjà repérés, d'introduire une série de déterminants relatifs à la gouvernance.

\subsection{La gouvernance comme déterminant de la performance des pôles à dominante PME}

Les travaux récents sur les agglomérations de PME s'attachent à établir un lien entre leur gouvernance et leur performance. En effet, ces agglomérations sont par nature des formes spécifiques dont la performance ne peut résulter d'un processus de coordination spontané. Quelle que soit la forme étudiée (district, cluster, pôle), ces formes sont marquées par (Mendez et Bardet, infra) :

- Une forte hétérogénéité ${ }^{3}$ des membres quant à leur nature et à leurs objectifs (coexistence d'acteurs individuels et collectifs, publics et privés);

- Des PME et TPE déficitaires en ressources financières, organisationnelles et stratégiques (Alberti, 2004), mais aussi et surtout en ressources de R\&D et en capacités de coopération ;

- Des PME et TPE qui adoptent des comportements individualistes, capables de se battre pour un nombre limité de ressources (travail, capital, services) conduisant ainsi à annuler les effets de proximité géographique ;

- Des membres qui s'inscrivent sur une trajectoire de développement local qui peut se traduire par des risques de polarisation ou de captage de ressources par un nombre limité d'institutions et d'entreprises ou d'exclusion pour d'autres.

La gouvernance est définie, dans cette perspective, comme un mode de régulation entre une pluralité d'acteurs (publics ou privés, insérés ou non dans des réseaux) en situation d'interaction selon différents modes (plus ou moins hiérarchiques, marchands, non marchands ou contractuels), à différentes échelles (du local à l'international). Cette gouvernance est appréhendée comme un déterminant de la performance au sens où elle participe à la création d'une dynamique collective entre des acteurs hétérogènes et déficitaires en ressources et en capacités d'interaction. Si la gouvernance est donc appréhendée comme un mode de régulation et de coordination entre acteurs, elle est le fait d'instances, de nature stratégique et/ou opérationnelle

Certains travaux récents ont cherché à différencier les formes prises par la gouvernance dans les pôles de compétitivité (Mendez et Bardet, infra ; Ehlinger et al. 2007), en particulier à

\footnotetext{
${ }^{3}$ Cette caractéristique ne doit pas être confondue avec la notion de diversité des membres, mobilisée en partie 1. La notion de diversité est ici plus large et permet de différencier les entreprises selon leurs caractéristiques individuelles : taille, structure du capital, activité principale, etc. (Tableau 1)
} 
travers l'analyse des différents modes. Dans la lignée d'Alberti (2004), Ehlinger et al. (2007) distinguent trois modes de gouvernance :

- Une gouvernance par la firme focale (encore appelé le pivot), qui régule les transactions au sein du réseau et agit comme un centre stratégique élargi : ce mode, assez éloigné du cluster «à la Porter», peut être observé dans certains pôles de compétitivité au sein desquels il existe une entreprise prédominante autour de laquelle se structure le pôle (cf. notamment PASS, Bocquet et al., 2008) ;

- Une gouvernance associative : cette «combinaison d'institutions privées et spécialisées en charge de l'élaboration, de l'adaptation et de l'exécution des règles collectives » (Ehlinger et al., 2007, p. 164), courante dans les districts industriels (au travers des comités de district), répartit la fonction de pilotage et de régulation entre les membres. Alberti (2001) souligne que l'absence d'un tel centre gouvernant constitue une faiblesse majeure pour certains districts ;

- Une gouvernance territoriale : ce mode est défini par Gilly et Wallet (2001) comme un processus de confrontation et d'ajustement de systèmes de représentations et d'actions de groupes d'acteurs proches géographiquement mais pouvant être issus de champs organisationnels et institutionnels différents. La gouvernance territoriale répond donc à la nécessité d'intégrer la diversité des acteurs en présence, en donnant aux acteurs publics un rôle clé «d'agents de coordination, d'arbitrage ou de communication » (Ehlinger et al., 2007, p. 165).

La principale différence entre les deux derniers modes repose sur la formalisation de l'instance de gouvernance en lien avec le type de performance attendue. La gouvernance associative s'avèrerait insuffisante pour assurer une dynamique collective à long terme dans la mesure où elle intègre mal la diversité des acteurs et la complexité de leurs relations. La viabilité et la performance dépendraient de la formalisation des liens entre les parties prenantes internes, externes et l'instance de gouvernance, cette formalisation faisant appel à trois dimensions :

- l'instance de gouvernance doit être représentative et légitimée par les divers acteurs internes (individuels et collectifs) pour être dotée d'une délégation de leurs pouvoirs de décision. Cette légitimation (en tant que processus d'acquisition d'une légitimité du point de vue des parties prenantes) sera facilitée par la présence de mécanismes sociaux de régulation tels que la confiance, la réciprocité ou la solidarité ;

- cette instance doit être dotée de ressources propres suffisantes (humaines, organisationnelles et financières) pour lui donner les moyens incitatifs ou coercitifs d'assurer ses rôles d'orientation stratégique, de services et de contrôle (Alberti, 2001); 
- la forme prise par l'instance de gouvernance dépend étroitement de facteurs de contingence internes (cycle de vie du pôle, individualisme des membre etc.) et externes ${ }^{4}$ (variables macroéconomiques, type d'industrie, intensité concurrentielle, etc.). Ceci suggère l'importance de la capacité d'adaptation de la structure elle-même pour la performance de l'ensemble à long terme.

La gouvernance territoriale, caractéristique d'une formalisation plus poussée, évite tout risque de lock-in ${ }^{5}$ et de résistance au changement. Elle constituerait une forme adaptée aux pôles de compétitivité dont la gouvernance doit répondre à des enjeux d'ordre à la fois public et local.

Nous cherchons ci-après à positionner un pôle de compétitivité de la région RhôneAlpes, Arve-Industries Haute-Savoie Mont-Blanc grâce aux critères de distinction identifiés dans cette partie théorique, à savoir la diversité des membres, les barrières à l'entrée et à la sortie, les modes de coordination et les différents types de performance attendue. Cette analyse nous conduira à discuter du type de gouvernance adéquate, selon la position du pôle par rapport aux deux idéaux-types.

\section{Le cas des pôles à forte dominante PME : l'exemple d'Arve-Industries}

Après une description des caractéristiques du pôle Arve-Industries qui nous permet de le positionner comme une forme «hybride» entre district et cluster, nous discutons de la forme de gouvernance qui serait appropriée pour fournir des éléments réponses aux problèmes et aux risques associés.

\subsection{Description et spécificités du pôle Arve-Industries}

Le pôle Arve-Industries Haute-Savoie Mont-Blanc regroupe, au 25 septembre 2008, 208 entreprises adhérentes, dont 90\% de PME, essentiellement de sous-traitance industrielle. Le pôle regroupe 28 laboratoires publics, 30 laboratoires privés, 12 centres de formation technique, 14 acteurs territoriaux et 12 communes. Les principaux secteurs clients sont l'automobile, l'aéronautique, les télécoms, le médical, le luxe et la domotique. Le pôle concerne essentiellement (en nombre d'adhérents) la zone géographique du Système Productif Local (SPL) de la Vallée de l'Arve (Technic Valley) - même s'il accueille aussi des entreprises situées dans tout le territoire départemental spécialisées dans les mêmes métiers. Le SPL de la vallée de l'Arve regroupe 800 PME de sous-traitance de mécanique, dont plus de 500 sont spécialisées

\footnotetext{
${ }^{4} \mathrm{Cf}$. Alberti (2001) pour la liste complète de ces facteurs internes et externes.

${ }^{5}$ La notion de lock in traduit la dépendance d'une organisation qui serait dans l'impossibilité de se tourner ailleurs (en dehors du territoire) sans encourir des coûts de changement substantiels.
} 
dans le décolletage. Si ce SPL a été initié en janvier 1999 par le maire de Cluses ${ }^{6}$, il est l'héritage d'un système productif horloger datant du XIXème siècle qui a évolué en district industriel du décolletage.

Les membres de la gouvernance du pôle ont une stratégie visant à accompagner par l'innovation la mutation des entreprises du décolletage vers la mécatronique. «Le pôle a pour ambition de faire évoluer la filière mécanique vers la fabrication d'ensembles multitechnologiques à forte valeur ajoutée en stimulant les partenariats entre centres de recherche, de formation, acteurs économiques et les entreprises afin de rester compétitif et innovant ${ }^{7}$.

\section{Méthodologie}

Nous avons conduit une recherche exploratoire entre janvier et mars 2008 sur le pôle ArveIndustries qui avait pour but d'identifier les critères d'évaluation de la performance par les acteurs de la gouvernance du pôle, ainsi que les déterminants de cette performance.

Nous avons interrogé, au cours d'entretiens approfondis, 19 personnes (soit 28 entretiens, certaines personnes, notamment du comité de direction, ayant été interrogées et rencontrées plusieurs fois) : l'ensemble des membres du comité de direction ( 7 personnes), des membres du bureau exécutif et de la présidence (7) et des chefs de projet (5). Cette phase qualitative s'est appuyée sur des entretiens semi-directifs de deux heures environ en face à face.

Pour des motifs de confidentialité, la liste des interviewés ne peut être communiquée. Tous les entretiens ont fait l'objet d'un enregistrement (même si certains acteurs ont demandé de couper l'enregistrement à certains moments pour pouvoir s'exprimer plus librement). Les entretiens ont fait l'objet d'un codage thématique sur les principales catégories suivantes issues de la littérature théorique: émergence du pôle, caractéristiques, SPL, performance (nature et facteurs explicatifs), gouvernance (CA/bureau/comité de direction), rôle, spécificités d'Arve-Industries, PME, sous-traitance. Les entretiens ont par ailleurs tous fait l'objet de compte-rendus détaillés, qui ont été validés par l'ensemble des acteurs. Des données secondaires ont été utilisées : dossier d'évaluation du pôle, extraits de presse sur les pôles, sites Internet du gouvernement et de différents pôles, articles scientifiques et communications à des colloques, à des journées de recherche, aux observatoires de l'innovation et des pôles/SPL. Une triangulation des données primaires et secondaires a été effectuée pour faire ressortir les principales caractéristiques du pôle et ses modes de fonctionnement.

Le lecteur trouvera ci-après la liste des sigles et abréviations utilisées :

- CTDEC : Centre Technique de l'industrie du Décolletage

- CETIM : CEntre Technique des Industries Mécaniques

- AED : Agence Economique Départementale de Haute-Savoie

- SNDEC : Syndicat National du Décolletage

- THESAME : Centre Européen d'Entreprise et d'Innovation

- CCI : Chambre de Commerce et d'Industries de Haute-Savoie

- C4i : Centre de Conception en Circuits Intégrés

\subsection{Mesures et déterminants de la performance d'Arve-Industries}

La confrontation de notre grille d'analyse empirique à Arve-Industries est menée en deux temps.

Le premier cherche à évaluer la «distance » de ce pôle par rapport aux deux idéaux-types de

\footnotetext{
${ }^{6}$ Cf. site http///www.diact.gouv.fr (consulté le 15/09/2008)

${ }^{7}$ Cf. site http://www.arve-industries.fr/?lang=fr\# (consulté le 15/09/2008)
} 
performance que sont le district industriel et le cluster. Cette distance est appréciée à la fois au regard des principaux déterminants retenus (diversité de ses membres, barrières à l'entrée et à la sortie, modes de coordination) et du type de performance attendue. Dans un second temps, nous identifions le rôle spécifique que peut jouer la gouvernance sur sa performance.

\subsubsection{Arve-Industries: plus proche d'un district ou d'un cluster ?}

Diversité des membres: Arve-Industries présente, comme tous les pôles français (de par l'exigence des conditions de création d'un pôle édictée par le gouvernement), une forte hétérogénéité des membres : entreprises, centres de recherche, centres de formation et autres partenaires (institutionnels, collectivités territoriales, centres techniques, etc.). L'implication des acteurs territoriaux est forte, dans la gouvernance ainsi que dans les projets, notamment de Thésame, du CTDEC, du CETIM, de l'AED, de la Chambre Syndicale de la Métallurgie, du SNDEC, de la CCI et de l'Université de Savoie. En revanche, la diversité s'avère moyenne en ce qui concerne la taille des membres du collège «industriels » et le type d'activité : le pôle est essentiellement composé de PME (à 90\%) en nombre d'adhérents; il comporte cependant quelques entreprises leaders sur leur marché, mais de taille moyenne. La diversité est toutefois renforcée par la présence de partenaires localisés hors des frontières géographiques ou sectorielles: laboratoires de recherche (CERN, CEA Leti, Ecole Polytechnique Fédérale de Lausanne, ...) et entreprises de taille mondiale (comme Schneider Electric, BioMérieux, Total). Les PME industrielles, surtout de sous-traitance mécanique, sont fortement concentrées dans le décolletage et la mécatronique. Les marchés cibles tendent à se diversifier: si l'automobile représente encore $60 \%$ de l'activité, les entreprises s'ouvrent vers de nouveaux marchés à plus forte valeur ajoutée comme l'aéronautique, la connectique et le médical.

Barrières à l'entrée et à la sortie : Les barrières à l'entrée sont a priori faibles. Le montant du « ticket d'entrée » dans le pôle (de 100 à 1000 euros selon la taille) garantit la possibilité à toutes les entreprises de devenir membres (y compris les TPE) dès lors qu'elles se situent dans le zonage $R \& D$ et dans les activités du pôle. Il convient de souligner que les barrières à l'entrée ont été affaiblies sous l'action des membres de la gouvernance. Face au risque de lock-in, ils ont choisi de créer la notion de «partenaire associé » permettant ainsi d'intégrer des membres distants (en dehors du zonage R\&D) pour favoriser l'accès à de nouvelles connaissances. De l'avis des acteurs, il n'existe pas non plus de barrières à la sortie. Notons que seules trois entreprises ont quitté le pôle.

Modes de coordination : Le pôle s'inscrit en continuité/rupture d'une logique territoriale préexistante (autour d'un SPL) marquée par une fort enracinement local, un héritage industriel 
d'initiative privée et une culture du secret et du cloisonnement. Si le SPL a permis la mise en commun de moyens autour d'une plate-forme locale, celle-ci n'avait pas débouché sur des projets collaboratifs :

"Le SPL n'avait pas une véritable activité envers les entreprises. Celui-ci était peu connu, ne gérait pas de projet collaboratif et ne fournissait pas de soutien. C'était plus une plate-forme qui gérait quelques crédits de la Région » (membre du comité de direction)

«La mise en commun de moyens pour atteindre des objectifs n'était donc pas une idée nouvelle mais la gouvernance et l'introduction d'industriels dans la gouvernance et les projets, ça, c'est nouveau! » (membre du comité de direction).

En revanche, le pôle a bénéficié de l'existence préalable de trois plates-formes technologiques (le centre productique Haute-Savoie, le C4i et le CTDEC) et de Thésame (dès 2000), qui avaient permis de mettre en réseau différents organismes (entreprises, institutions locales, universités et centres de recherche). La création du pôle a amplifié un rééquilibrage territorial au niveau du département, une ouverture européenne et a insufflé une nouvelle dynamique d'échanges entre, d'une part, des entreprises qui ne se côtoyaient pas et, d'autre part, différents acteurs publics et privés qui n'avaient pas l'habitude de collaborer. Dans le pôle, la coopération est essentiellement organisée par les institutions publiques, acteurs clés de la gouvernance. La majorité des acteurs reconnaissent toutefois qu'il reste des progrès à faire pour impliquer les partenaires mais qu'il y a de «vrais projets, de vraies thématiques »(chargé de mission).

\section{Performance :}

- Performance territoriale : les critères de performance au niveau du territoire ne diffèrent pas entre le district et le cluster et on les retrouve pour le pôle Arve-Indutries. Dans le département de Haute-Savoie, l'industrie, représentant 30\% du PIB et de l'emploi, est un moteur. Par ailleurs, d'autres secteurs d'activité, comme la construction, vivent grâce à la présence forte de l'industrie. Dans ce contexte, le développement économique et l'emploi apparaissent comme les critères principaux de performance cités par les répondants. L'emploi, c'est non seulement la création d'emplois, mais aussi l'évolution du niveau de qualification des salariés :

"L'emploi est le but final du pôle » (responsable projet).

"La performance du pôle doit se mesurer au moins au maintien de l'industrie en Haute-Savoie et, au mieux, à son développement » (membre du comité de direction).

"A long terme, à quoi sert un pôle ?A créer des emplois : le nombre et la qualité des emplois sont de vrais indicateurs» (membre du comité de direction).

Toutefois, deux ans d'existence ne suffisent pas, certaines personnes interrogées allant même jusqu'à dire que l'évaluation de la performance d'un pôle est difficile à réaliser :

"La performance? Il faudra la mesurer. L'évaluation des pôles par le gouvernement est arrivée beaucoup trop tôt car les effets ne sont pas encore visibles » (membre du bureau exécutif) 
Mais une minorité soulève les limites d'un tel discours, rejoignant ainsi l'importance du rôle stratégique que doit jouer la gouvernance dans l'évaluation de la performance d'un pôle :

"Il faut cependant se méfier d'un recours systématique à cette façon de voir qui pourrait masquer une absence réelle de résultats : il faut trouver des indicateurs tangibles!» (membre du Bureau)

D'autres critères macroéconomiques sont mentionnés, notamment la balance commerciale du pôle, la croissance du tissu industriel vers une position plus «robuste », la santé financière des entreprises membres, leur taux d'exportation, le développement d'activités, le développement économique, la pérennité des emplois, l'attractivité/l'image... Ces indicateurs de résultat sont le reflet d'une performance qui se mesure par une dynamique (attractivité, croissance, etc.) :

- Performance collective : les effets positifs liés à l'agglomération spatiale des activités sont peu mentionnés. Encore peu visibles du fait de la jeunesse du pôle, ils sont néanmoins au cœur de l'action stratégique du comité de direction dans le cadre de projets dits structurants. Des premiers symptômes positifs apparaissent. Ils se concrétisent notamment par des effets de diffusion vers des activités complémentaires telles que le traitement de surface et les services industriels de sous-traitance de proximité à la disposition des entreprises de mécanique. En revanche, les déséconomies liées à la proximité et à la «concurrence » de la Suisse sont nettement affirmées. Elles se traduisent par une pénurie de main d'œuvre et une réelle difficulté à recruter du personnel qualifié. Un projet collaboratif en ressources humaines de grande ampleur (Talents $2010^{8}$ ) a été mis en oeuvre pour trouver des solutions à ce problème majeur. En ce qui concerne les externalités de communication, les propos recueillis indiquent la présence d'une génération plus ouverte au partage et au tissage de liens- même si l'individualisme reste fort :

"Il faut retenir la volonté qu'ont tous les acteurs de travailler en réseau. Tous doivent partager une vision commune du pôle et tous doivent jouer le jeu (abandonner les prérogatives individuelles) pour qu'il perdure » (responsable de projet).

Ce qui est mis en avant, c'est surtout la dynamique coopérative qui s'est créée grâce au pôle et qui permet d'avoir des financements publics pour des projets collaboratifs. Cette dynamique peut certes être appréhendée au travers de l'évolution du nombre d'adhérents au pôle. Toutefois, il nous semble plus pertinent de la mesurer par l'évolution du nombre d'entreprises impliquées

\footnotetext{
${ }^{8}$ Talents 2010 est une action transversale dont l'objectif est de disposer durablement d'une main d'œuvre qualifiée en fonction des besoins des entreprises. Le projet se découpe en 3 volets: Image et promotion des métiers, Gestion prévisionnelle des Emplois et des Compétences (GPEC) et gestion des Ressources Humaines (http://www.arve-industries.fr/talents_2010-fr148.html, consulté le 15/09/2008)
} 
et actives au sein des projets, quelle que soit leur nature (R\&D ou non R\&D). Le tableau 2 fait apparaître que la proportion de membres actifs est stable (environ 50\%) et que le nombre d'actifs augmente donc proportionnellement au nombre d'adhérents.

\section{Tableau 2 ici}

Les acteurs insistent aussi sur la réalité de la collaboration entre les acteurs institutionnels de l'économie permettant aux entreprises d'avoir des retombées effectives. Le nombre de partenaires institutionnels impliqués est d'ailleurs cité comme un critère de performance :

"Actuellement, tout le monde est dedans, et pas contraint et forcé mais enthousiaste. Personne n'est laissé sur le bord de la route » (membre du bureau exécutif)

Au niveau collectif, ce sont les programmes et les projets qui font l'objet d'une évaluation de la performance. C'est sur les projets technologiques au sein des trois programmes sur l'innovation que cette évaluation de la performance va se concrétiser :

«La réussite, elle va se mesurer rapidement... sur les projets technologiques » (membre du bureau exécutif)

Deux sortes de critères co-existent pour les acteurs et varient selon leur rôle et leur fonction : plus orientés vers la recherche pour les responsables de projets d'innovation, plus orientés vers les retombées pour les adhérents et le nombre d'adhérents pour les partenaires institutionnels départementaux notamment (cf. tableau 3).

\section{Tableau 3 ici}

Les acteurs interrogés insistent sur le fait que les critères d'évaluation de la performance dépendent également du type de pôle :

"Un pôle mondial doit faire émerger énormément de brevets $R \& D$ en optimisant au maximum les ressources publiques. Pour des pôles toiles ou territoriaux, dont fait partie Arve-Industries, un critère serait d'amener un maximum d'entreprises à un niveau «best in class». Il est trop tôt pour le mesurer» (membre du comité de direction)

- Performance individuelle : la performance pour une entreprise s'évalue non seulement en termes d'économies de coûts, mais aussi - et surtout - d'accroissement de son potentiel et de ses capacités d'innovation. Les entreprises ont pour particularité d'être plutôt orientées vers l'innovation de process et l'innovation organisationnelle que vers l'innovation produit. Ceci peut s'expliquer de par la nature de ses membres (PME de sous-traitance mécanique et décolletage). Les projets de $\mathrm{R} \& \mathrm{D}$ «hard» (coupe, usinage, etc.) coexistent avec des projets «soft» (veille économique, innovation organisationnelle ou ressources humaines). Pour les projets de $R \& D$, le pôle s'insère d'ailleurs dans la continuité des efforts entrepris pour protéger les inventions des entreprises, y compris celles des les plus petites, les incitant ainsi à innover. 
L’ensemble des éléments qui précèdent est récapitulé dans le tableau 4. Le pôle ArveIndustries se situe donc à mi-chemin entre district et cluster, avec des spécificités de chacun de ses deux idéaux-types. Quel est l'impact de cette position intermédiaire hybride sur les modalités de la gouvernance?

\section{$\underline{\text { Tableau } 4 \text { ici }}$}

\section{2.b. L’importance de la gouvernance pour un pôle de PME}

La gouvernance d'Arve-Industries est aujourd'hui composée :

- d'une gouvernance stratégique. Le Conseil d'Administration comprend 60 membres qui se réunissent 2 fois par an en Assemblée Générale. Le Bureau Exécutif, élu par l'Assemblée Générale, est chargé de la définition de la stratégie et des grands objectifs. Il est composé de 17 membres qui se réunissent tous les 2 à 3 mois. Les décisions au niveau du CA sont soumises au vote à la majorité compte-tenu du nombre de membres (60) ;

- d'une gouvernance opérationnelle. Le Comité de Direction est composé de 7 membres9 se réunissant toutes les 2 semaines environ. Il assure le pilotage à travers une approche transversale par processus: intelligence économique, labellisation de nouveaux projets, marketing de l'innovation, conduite stratégique. Il réalise l'évaluation des retombées des projets, leur reporting, les relations extérieures et la gestion au quotidien. Pour chaque programme, il y a des grappes de projets avec un comité de pilotage mixte comprenant un chef de projet, des industriels, des experts, des chercheurs et/ou des institutionnels. Les décisions du comité de direction se prennent par consensus.

Pour évaluer le rôle que joue la gouvernance sur la performance du pôle, nous étudions sa représentativité et sa légitimité, le niveau des ressources dont elle dispose et sa capacité d'adaptation vis-à-vis des évolutions de son contexte interne et externe. En matière de gouvernance, l'Etat n'a pas fait de prescription dans le cahier des charges, même s'il a incité les pôles à mettre en place une instance de gouvernance propre:

"Chaque pôle s'est débrouillé selon son histoire et son contexte» (membre du comité de direction)

Au démarrage, la gouvernance opérationnelle était assurée par un groupe informel composé des partenaires fondateurs et par la seule salariée du pôle (sous le contrôle du Président). Ce fonctionnement a montré ses limites en l'absence de procédures de coordination définies et

\footnotetext{
${ }^{9}$ Soit, en plus du Président : Thierry Guillemin, Directeur du CTDEC, délégué général, Gisèle Bonnot, directrice de l'AED, déléguée à l'environnement économique, Jean Breton, Thésame, délégué à la coordination de projets, Diane Dubois, chargée de mission animation/communication, André Montaud, directeur de Thésame, délégué aux relations extérieurs, Laurent Tabourot, Université de Savoie, délégué aux relations universitaires.
} 
d'une décentralisation suffisante des pouvoirs de décision. C'est dans ce contexte que la gouvernance du pôle est passée du mode informel (associatif, selon la terminologie d'Ehlinger et al., 2007) à un mode plus directif avec la constitution d'un comité de direction en charge de la gouvernance opérationnelle. Celle-ci s'est traduite par une formalisation accrue et une plus forte ingérence des membres de la gouvernance dans les projets, contribuant ainsi à renforcer la dynamique collaborative. Comme le soulignent Defélix et al. (2006), les entreprises gèrent les projets avec leurs propres pratiques, ce qui provoque des vrais problèmes compte tenu de leur grande diversité. Le comité de direction avait la mission d'harmoniser la gestion des projets et d'introduire de réelles compétences en la matière (les PME de sous-traitance industrielle ne l'ayant pas souvent). Le choix de recourir à un comité de direction - et non à un directeur répond avant tout à un principe de légitimité :

«Il fallait quelqu'un capable de faire bouger l'ensemble - ce qui impliquait une forte légitimité de celui-ci. Quelqu'un qui aurait débarqué n'aurait pas pu (...) Il fallait un facilitateur capable de faire travailler les gens ensemble, un homme de fédération avec un peu de légitimité »(Membre du bureau exécutif)

Une autre raison qui a conduit à privilégier un Comité de Direction tient à la complémentarité des compétences ainsi qu'à la qualité des relations qu'entretiennent les membres du comité de direction :

"Nous avons choisi un Codir plutôt qu'un directeur de pôle car nous avons voulu regrouper des compétences existantes issues des plates-formes technologiques »(responsable de programme)

Les relations entre les membres de la gouvernance opérationnelle du pôle sont facilitées par une vision commune, et une culture orale et informelle. Ces relations sont anciennes, basées sur une confiance et des valeurs partagées autour d'une culture locale et de PME :

«Nous sommes un pôle travaillant comme des PME, avec des tensions très fortes. Nous avons le même vécu, c'est pour cela que les PME nous acceptent » (membre du comité de direction)

Le comité de direction a permis de passer un cap avec une organisation plus structurée sur la base d'une approche fonctionnelle. Si les modes de coordination étaient jusqu'à présent largement informels entre tous les membres de la gouvernance, les règles commencent à être codifiées sous l'impulsion du comité de direction :

"Les règles de fonctionnement sont en train d'être codifiées et devraient être communiquées aux chefs de projet avant la fin de l'année 2008 (...); il faut plaquer des modes de gouvernance sans relations hiérarchiques et trouver des modes de fonctionnement commun » (membre du comité de direction) 
Les autres difficultés sont liées à l'insuffisance des ressources humaines pour mener à bien le rôle de gouvernance opérationnelle et insuffler une véritable dynamique collaborative à long terme. D’une part, les membres du comité de direction sont saturés :

"On est dans une étape de professionnalisation et de déploiement organisationnel, mais on manque de main d'œuvre. Il faut renforcer les compétences (besoin de profils particuliers). On est à la limite du dispositif » (membre du comité de direction)

D'autre part, on note un manque de compétences de certains responsables de projets en matière de management de l'innovation, susceptible de freiner l'efficacité de certains projets en cours :

"Après l'euphorie de départ, il y a eu une surcharge de travail et le constat d'un certain nombre d'insuffisances, notamment une méconnaissance du management de l'innovation. Soit le pôle mourait, soit on trouvait une solution » (membre du comité de direction)

«L'apprentissage de la gestion de projet $R \& D$ a été un peu difficile. Qu'est-ce qu'il fallait faire? J'ai consulté de nombreux ouvrages sur le sujet pour revenir à du bon sens. L'expérience joue » (chef de projet).

Au total, à travers la ré-organisation en cours, la gouvernance du pôle fait preuve d'une certaine capacité d'adaptation à son contexte. Les acteurs que nous avons interrogé ont tous insisté sur la nécessaire prise en compte de la spécificité du pôle, notamment sa composante PME, qui influe sur le type de gouvernance :

«C'est toute la différence entre les pôles de grappes et les pôles de toile. Les pôles de grappes sont des pôles mondiaux très structurés autour de quelques leaders. Les pôles de toile reposent sur un maillage et la résistance dépend de l'intensité des liens entre les techniciens du territoire. Le Comité de direction est le moteur du pôle permettant une évolution des compétences de beaucoup de gens, même s'il sort de son rôle » (membre du comité de direction)

\section{Discussion et conclusion}

Notre recherche exploratoire fait ressortir que le pôle Arve-Industries présente les caractéristiques d'une forme organisationnelle hybride entre district et cluster. Le principal critère de performance réside dans la dynamique collective et collaborative qui ne peut naître, dans un pôle de PME, que sous l'impulsion d'une gouvernance forte. Le lien entre performance et gouvernance apparaît ici de manière explicite. Il se concrétise par la création d'un comité de direction qui, s'il a joué son rôle initial de coordination et de suivi opérationnel du pôle, et est à l'origine de nouveaux projets et de leur labellisation, se trouve aujourd'hui confronté à un besoin de renforcement. On voit ici un exemple non seulement de la prise de conscience de l'importance de l'instance de gouvernance pour le fonctionnement d'un pôle, mais aussi d'évolution de cette gouvernance. Ces instances étaient, en 2006, majoritairement des coquilles vides en cours de constitution ne mettant pas la priorité sur les RH (Defélix et al., 2006). La gouvernance associative (liée à l'implication de partenaires institutionnels), typique d'une régulation de district industriel, a joué son rôle en phase de création du pôle. Toutefois, la 
croissance de celui-ci et la nécessité d'afficher des performances répondant aux attentes multiples nécessite de passer à un autre type de gouvernance qui, tout en restant territoriale (Ehlinger et al., 2007), serait formalisée avec des moyens plus importants permettant de renforcer l'activité du pôle. En effet, les PME, on le voit, se trouvent confrontées à la pression du quotidien avec des difficultés à dégager du temps. Elles n'ont pas les ressources, financières, matérielles ou humaines, pour s'impliquer dans la gouvernance du pôle, ni parfois même dans la coordination des projets. Seuls les partenaires territoriaux institutionnels, à condition qu'ils soient légitimes, peuvent remplir une telle mission. Il est par ailleurs nécessaire, comme le réaffirment Mendez et Bardet (infra), d'appréhender la gouvernance en prenant en compte la nature du pôle.

Les résultats fournis par l'étude du pôle Arve-Industries nous permettent d'avancer que la gouvernance territoriale, i.e. avec une forte implication des acteurs institutionnels locaux, se présente comme un mode adapté aux pôles qui s'inscrivent dans la continuité d'un district industriel avec un fort ancrage historique et territorial tout en cherchant à introduire une dynamique d'innovation propre aux clusters à l'anglo-saxonne.

Nous nous insérons également dans la lignée des travaux de Defélix et al. (2006) qui mettent en avant qu'il n'y a pas de pôle de compétitivité efficace sans la dimension humaine, nécessaire à l'une instance de gouvernance. Les pratiques de GRH doivent apporter leur contribution au bon fonctionnement de la collaboration et de la synergie recherchées entre les partenaires. Ils se matérialisent aussi, dans le cas du pôle Arve-Industries, par la mise en place d'actions transversales spécifiques autour des RH, puisque Talents 2010 a aussi pour vocation de fédérer les entreprises autour des problèmes d'emploi, contribuant aussi à renforcer la dynamique coopérative.

Cette conception de la gouvernance territoriale et de l'implication institutionnelle présente, selon nous, deux types d'avantages. D'une part, en préservant l'autonomie d'interaction entre les groupes d'acteurs au sein et au delà du territoire, elle établit une rupture nette avec le modèle «localisé » de la performance d'un district en favorisant l'ouverture sur l'extérieur. La création du statut de «partenaire associé » fournit un exemple caractéristique de cette volonté d'ouverture au delà d'une logique strictement territoriale. Elle est, de ce point de vue, compatible avec la conception fournie « en creux » par Porter. D'autre part, elle ne tombe pas dans le piège du one best way selon lequel il existerait une structure unique capable de soutenir a priori un type de performance donné (i.e. l'emploi, dans les districts). Prendre en compte la complexité des relations et leur nature dynamique implique la coexistence d'une variété de structures formelles dont les performances attendues peuvent être variables selon le 
type d'acteurs et de parties prenantes concernées. Cette variété est renforcée par le rôle décisif que peuvent jouer les facteurs de contingence internes et externes sur les structures elles-mêmes (comme la proximité avec la Suisse dans le cas d'Arve-Industries) - et l'aptitude collective des acteurs à y faire face. La gouvernance est de nature à garantir une performance collective comme élément moteur de la performance tant territoriale qu'individuelle. A ce titre, l'implication des institutions et des plates-formes technologiques locales dans la gouvernance des pôles à forte dominante PME s'avère cruciale.

Comme toute recherche, celle-ci n'est pas exempte de limites. Il est en particulier difficile de travailler sur la notion de performance sans pouvoir bénéficier de mesures objectives de cette performance. Nous avons tenté de pallier cette limite en fournissant les critères cités par les répondants de la performance individuelle et collective. Toutefois, nous sommes conscientes du fait que, si nous avions interrogé les entreprises adhérentes, d'autres critères seraient apparus, comme la pérennité ou la préservation de l'indépendance, par exemple, pour les PME n'appartenant pas à un groupe industriel (qui représentent la majorité des adhérents d'Arve).

Des recherches complémentaires seraient à mener sur le lien entre gouvernance (du pôle et des projets) et performance (collective et individuelle) et sur l'implication des institutions territoriales dans un modèle «hybride» entre district et cluster pour les pôles de PME. Des comparaisons sur un large échantillon de pôles de PME pourraient être conduites sur les ressources propres du pôle, la structure formelle de la gouvernance, sa légitimité, sa représentativité (de la diversité des membres) et sa capacité tant d'ouverture que d'évolution.

Cette analyse a été menée en phase de lancement du pôle de compétitivité ArveIndustries et devrait être poursuivie afin de pouvoir observer et évaluer la performance à moyen terme en lien avec les nouvelles reconfigurations des modes de gouvernance en cours. Par ailleurs, les objectifs de la gouvernance sont clairement de provoquer des retombées de compétences sur les entreprises du pôle. Ceci se traduit notamment par l' "inclusion », grâce au statut de "partenaire associé », d'entreprises de renommée mondiale très innovantes, situées loin des frontières géographiques du pôle, au sein des projets collaboratifs. A l'évidence, ce type de retombées ne peut être observé aujourd'hui, ce qui milite pour des recherches futures de nature dynamique et longitudinale. 
Tableau 1 : Principaux déterminants et nature de la performance dans le district industriel et le cluster

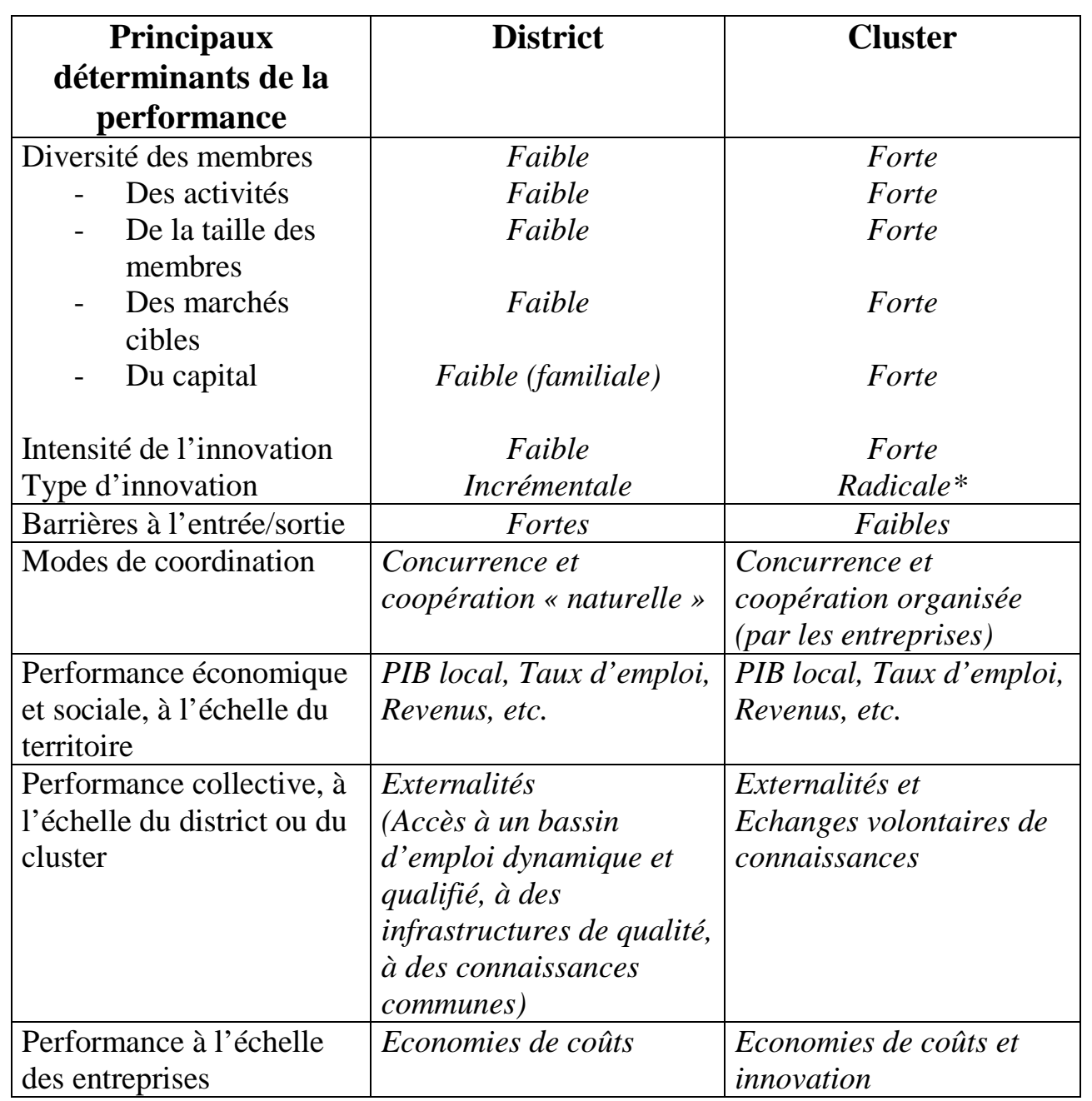

* La différence entre innovations incrémentale et radicale est conforme à celle retenue par l'enquête communautaire sur l'innovation (OCDE, 1997). Elle repose essentiellement sur le degré de nouveauté de l'innovation introduite par l'entreprise considérée. L'innovation incrémentale implique un produit ou un procédé «nouveau pour l'entreprise » uniquement. L’innovation radicale implique un produit ou un procédé « nouveau pour l'industrie ». 
Tableau 2 : Evolution du nombre d'adhérents et d'entreprises actives

\begin{tabular}{|c|c|c|c|}
\hline & $\begin{array}{c}\text { Au 30/09/2006 } \\
\text { (sources internes) }\end{array}$ & $\begin{array}{c}\text { Au 30/09/2007 } \\
\text { (sources internes) }\end{array}$ & Au 30/09/2008*** \\
\hline $\begin{array}{c}\text { Nombre } \\
\text { d'adhérents* }\end{array}$ & 128 & 177 & 208 \\
\hline $\begin{array}{c}\% \text { d'adhérents } \\
\text { actifs** }\end{array}$ & $50 \%$ & $50 \%$ & $50 \%$ \\
\hline $\begin{array}{c}\% \text { d'actifs dans } \\
\text { les projets R\&D }\end{array}$ & $25 \%$ & $25 \%$ & $22 \% * * * *$ \\
\hline
\end{tabular}

Source : Arve-Industries

* Le nombre d'adhérents comprend les entreprises répondant aux critères de zonage définis par le gouvernement et ayant payé leur cotisation au pôle (hors entreprises partenaires)

** Actifs dans les projets ou actions du pôle

*** http://www.arve-industries.fr/ (consulté le 25/09/2008)

**** Soit 45 entreprises, la baisse s'expliquant par la diminution du nombre de nouveaux projets en 2008.

Tableau 3. Critères de performance des projets cités par les répondants

\begin{tabular}{|l|l|}
\hline Critères quantitatifs & Critères les plus cités \\
\hline & $\begin{array}{l}\text { Nombre de projets d'innovation, de brevets, d'innovations réelles } \\
\text { (produits), Nombre et croissance des adhérents, Nombre de chercheurs } \\
\text { travaillant dans l'entreprise, Nombre de chercheurs dans les organes de } \\
\text { décision des entreprises, Nombre de partenariats avec des laboratoires, } \\
\text { Augmentation du budget d'innovation des entreprises }\end{array}$ \\
\hline Critères qualitatifs & $\begin{array}{l}\text { Respect des dates butoirs (délivrables) pour les projets d'innovation, } \\
\text { retour des entreprises et des médias, synergies et opérations communes } \\
\text { en lien avec les institutions du territoire, degré d'implication des } \\
\text { entreprises dans les projets d'innovation (comité de pilotage ou groupes } \\
\text { de travail), taux de participation aux réunions d'informations, nature de } \\
\text { leurs réponses aux enquêtes du pôle. }\end{array}$ \\
\hline
\end{tabular}


Tableau 4 : Positionnement du pôle Arve-Industries entre district et cluster

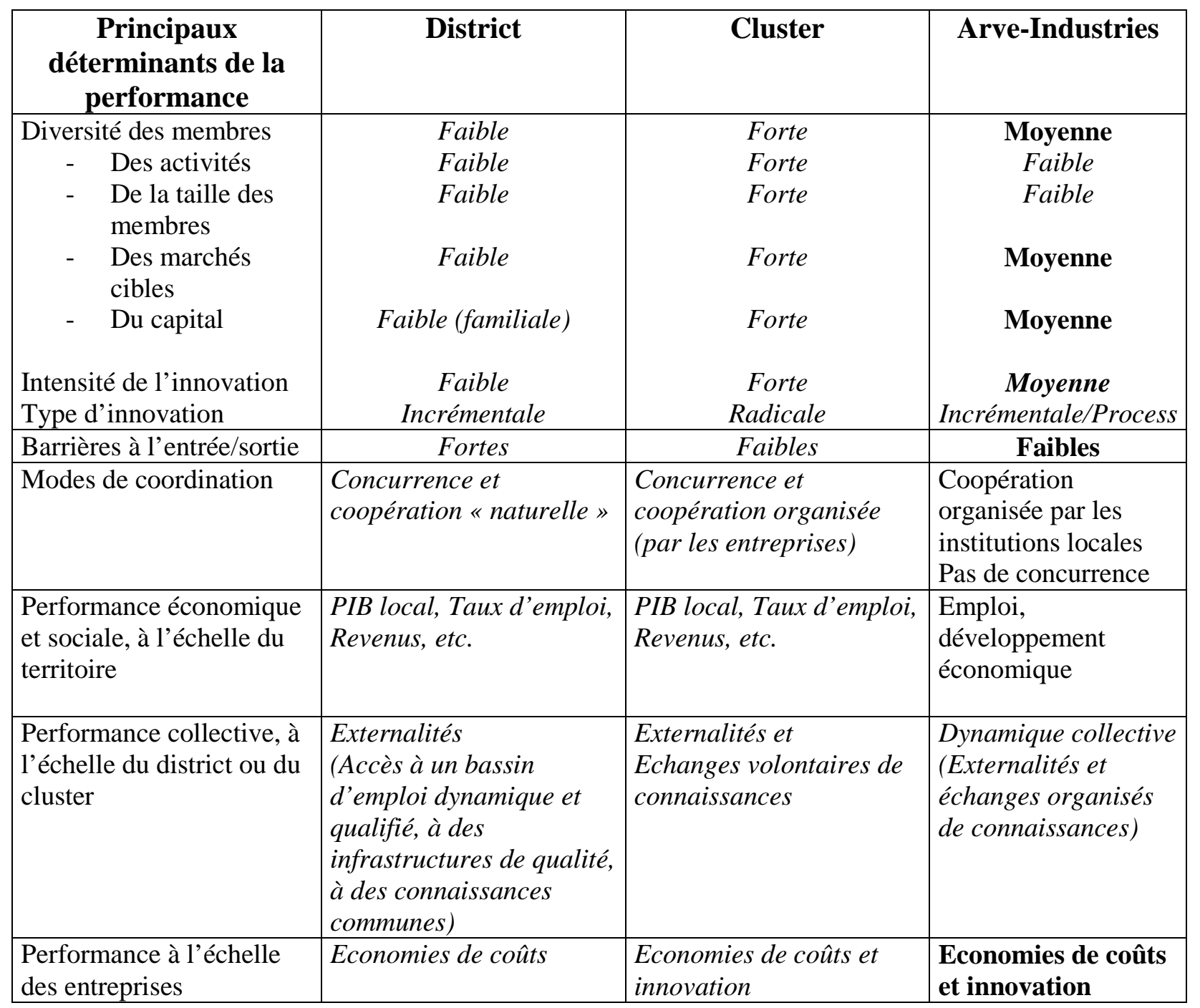




\section{Bibliographie}

Alberti F., The Governance of Industrial Districts: a Theoretical Footing Proposal, Liuc Papers, $\mathrm{n}^{\circ}$ 82, Serie Piccola e Media Impresa 5, 2001.

Alberti F., The Crisis of the Industrial District of Como: A Longitudinal Analysis of Its Evolution, 20th Egos Colloquium, juillet, Ljubljana, 2004.

Antonelli C., Collective Knowledge Communication and Innovation: the Evidence of Technological Districts, Regional Studies, vol. 34, n 6, p. 535-547, 2000.

Becattini G., Le district industriel : milieu créatif, Espace et Société, nº66-67, p.147-64, 1981.

Bocquet R., Mendez A. et Mothe C., Pôles de compétitivité et PME : quelles spécificités ?, Etats Généraux du Management, octobre, Paris, 2008.

Calmé I., Chabaud D., Les pôles de compétitivité : renouvellement ou continuité dans l'étude des systèmes territorialisés ?, AIMS, Montréal, juin, 2007.

Carluer F., Réseaux d'entreprises et dynamiques territoriales: une analyse stratégique, Géographie Economie et Société, vol. 8, n², p. 193-214.

Carpinetti LCR., Gerolamo MC., Galdámez EVC., Continuous Innovation and Performance Management of SME Clusters, Creativity and Innovation Management, vol. 16, $\mathrm{n}^{\circ} 4, \mathrm{p}$. 376-385, 2007.

Defélix C., Culié J.D., Retour D. et Valette A., Les pôles de compétitivité, laboratoires d'innovation en ressources humaines?, Revue Française de Gestion Industrielle, vol. 15, n³, p. 69-86, 2006.

Ehlinger S., Perret V., Chabaud D., Quelle gouvernance pour les réseaux territorialisés d'organisations ?, Revue Française de Gestion, n¹70, p.155-171, 2007.

Gilly J.P, Wallet F., Forms of proximity, Local Governance and the Dynamics of Local Economic Spaces : The case of Industrial Conversion Processes, International Journal of Urban and Regional Research, vol. 25, n³, p.553-570, 2001.

Grando, A., Belvedere V., District's Manufacturing Performances: A Comparison Among Large, Small-to-Medium-sized and District Enterprises, International Journal of Production Economics, vol. 104, n 1, p. 85-99, 2005.

Grossetti M., Concentration d'entreprises et innovation : esquisse d'une typologie des systèmes productifs locaux, Géographie, Économie, Société, vol.6, p.163-177, 2004.

Gundolf K., Jaouen A., Temri L., Le comportement d'innovation des PME dans les pôles de compétitivité : un cadre d'analyse, Colloque du PESOR, mars, Faculté Jean Monnet, 2007. 
Martin, R., Sunley, P., Deconstructing Clusters: Chaotic Concept or Panacea?, Journal of Economic Geography, vol. 3, p. 5-35, 2003.

Martin R., Sunley P., Complexity Thinking and Evolutionary Economic Geography, Journal of Economic Geography, vol. 7, n 5, p. 573-601, 2007.

Mendez A., Bardet M., Quels systèmes de gouvernance pour les pôles de compétitivité constitués de PME : entre logiques d'intégration et de différenciation, Revue Française de Gestion, infra, 2009.

Moulaert F., Sekia F., Territorial Innovation Models: A critical survey, Regional Studies, vol.37, n³, p.289-302, 2003.

OCDE, Manuel d'Oslo, Principes directeurs pour le recueil et l'interprétation des données sur l'innovation, 3e édition, Eurostat, éd. OCDE, 1997.

Porter M., Location, Competition and Economic Development: Local Clusters in a Global Economy, Economic Development Quarterly, Vol. 14, No. 1, p. 15-34, 2000.

Porter M., Competitiveness in Rural U.S. Regions: Learning and Research Agenda, Institute for Strategy and Competitiveness, Harvard Business School, Harvard, 2004.

Tallman S., Jenkins M., Henry N., Pinch S., Knowledge, Clusters, and Competitive Advantage, Academy of Management Review, vol. 29, n² 2, p. 258-271, 2004.

Torre A., Rallet A., Proximity and Localization, Regional Studies, vol. 39, n 1, p. 47-60, 2005. 